\title{
THE SOLUTIONS OF SECOND ORDER LINEAR ORDINARY DIFFERENTIAL EQUATIONS ABOUT A TURNING POINT OF ORDER TWO( ${ }^{(1)}$
}

\author{
BY \\ ROBERT W. MCKELVEY
}

1. Introduction. This paper is concerned with the problem of describing, for large values of a complex parameter $\lambda$, the behavior of the solutions of a class of differential equations of the form

$$
d^{2} u / d x^{2}-\left[\lambda^{2} P_{0}(x)+\lambda P_{1}(x)+Q(x, \lambda)\right] u=0 .
$$

This equation is considered over a real interval $a \leqq x \leqq b$ which contains a second order zero of $p_{0}(x)$. The coefficient $Q(x, \lambda)$ is of the form

$$
Q(x, \lambda)=\sum_{j=0}^{\infty} q_{j}(x) / \lambda^{j}
$$

and $p_{0}(x), p_{1}(x)$, and the $q_{j}(x)$ 's are suitable restricted bounded functions. Specifically, formulas are sought which represent solutions of (1.1) in a certain asymptotic sense when $|\lambda|>N\left({ }^{2}\right)$.

Existing asymptotic theory of the equation (1.1) is known to depend upon the nature of the vanishing of $p_{0}(x)$ and $p_{1}(x)$, these being coefficients of positive powers of $\lambda$. A zero of $p_{0}(x)$ has been called a turning point of the equation, the order of the turning point being the order of the zero. In an interval where $p_{0}(x) \neq 0$, complete asymptotic expansions of a pair of solutions have long been known; this case was the subject of the classical work of Birkhoff [1]. A recent advance, due to R. E. Langer [3], has brought the theory of the simple turning point to a similar degree of completion. For higher order turning points, on the other hand, the theory is still fragmentary, being most highly developed for a turning point of order two. In this case expansions have in fact been given $[2 ; 4]$, but only the leading term of an expansion has been expressed in any simple way. For terms after the first, the known expression are extremely complicated, each successive term a degree more involved than the one before.

It is the object of the present paper to obtain expansions, valid in the presence of a second order turning point, whose terms to any order are simply expressed. An algorithm will be given which involves only simple power series

Presented to the Society, December 30, 1953; received by the editors June 10, 1954.

(1) From the author's University of Wisconsin thesis, under the direction of R. E. Langer. Research supported by the Office of Naval Research.

(2) $|\lambda|>N$ simply abbreviates " $\lambda$ sufficiently large"; consequently $N$ need not be the same in different expressions. 
computations and single quadratures, and which leads to formal solutions of equation (1.1). These formal solutions are then shown to represent true solutions in an asymptotic sense. The algorithm is patterned after one employed by R. E. Langer in his theory of the simple turning point, but necessarily differs from it since that theory does not generalize directly to the second order case. The central role of the Bessel functions in the first order theory is taken here by the confluent hypergeometric (Whittaker) functions.

The following specific assumptions are to be made concerning the (closed) interval $(a, b)$ and the coefficients $p_{0}(x), p_{1}(x)$, and $q_{j}(x)$ :

(i) $(a, b)$ contains precisely one point at which $p_{0}(x)$ vanishes, the zero being of the second order. For convenience it is assumed that $a<0<b$, and that the zero of $p_{0}(x)$ is located at the origin.

(ii) $p_{0}(x), p_{1}(x)$, and $q_{j}(x)$ are indefinitely differentiable on $(a, b)$ and are expansible in Maclaurin series in a neighborhood of the origin. The series (1.2) is uniformly convergent and termwise differentiable by $x$ on $(a, b)$ when $|\lambda|>N$.

A less essential hypothesis, made to permit a detailed yet reasonably brief discussion, is that

(iii) the coefficient $p_{0}(x)$ is real and non-negative on the interval $(a, b)$. $p_{1}(x)$ and $q_{j}(x)$ are in general complex.

2. The first approximating equation. With $p_{0}^{1 / 2}(x)$ understood to designate that root of the (non-negative) number $p_{0}(x)$ which has the sign of $x$ itself, let the functions $\phi(x), \xi$, and $\psi(x)$ be defined by the formulas

$$
\begin{aligned}
& \text { (a) } \phi(x)=p_{0}^{1 / 2}(x), \quad \text { (b) } \xi=2 \lambda \int_{0}^{x} \phi(s) d s, \\
& \text { (c) } \psi(x)=\left[\int_{0}^{x} \phi(s) d s\right]^{1 / 4} /[\phi(x)]^{1 / 2} .
\end{aligned}
$$

Observe that both $\phi(x)$ and its integral vanish at the origin, to the order of $x$ and $x^{2}$ respectively, but have no other zeroes on $(a, b)$. When $x>0$ these functions have the common argument zero, while for $x<0$ they are assigned in turn the argument values $\pi$ and $2 \pi$. The convention is to be adopted throughout that a quantity raised to a real power has its argument thereby multiplied by the power. With this convention $\psi(x)$ is seen to need only a suitable definition at the origin to become a real and positive function which is continuous, in fact indefinitely differentiable, on the interval $(a, b)$. It follows from the formulas that $\left(^{3}\right)$

$$
\frac{\xi^{1 / 2}}{\xi^{\prime}}=\frac{\psi^{2}}{(2 \lambda)^{1 / 2}}
$$

(3) $f^{\prime}(x, \lambda)$ means $d f(x, \lambda) / d x$. 
and, upon taking logarithms and differentiating, that

Consider the function

$$
\frac{\xi^{\prime \prime}}{\xi^{\prime}}+2 \frac{\psi^{\prime}}{\psi}=\frac{\xi^{\prime}}{2 \xi} \text {. }
$$

$$
v(x, \lambda)^{-}=\psi(x) \eta(\xi, \lambda)
$$

in which the factor $\eta(\xi, \lambda)$ is any solution of the differential equation

$$
\begin{array}{ll}
\text { (a) } \frac{d^{2} \eta}{d \xi^{2}}+\frac{1}{2 \xi} \frac{d \eta}{d \xi}-\left[\frac{1}{4}-\frac{k(\lambda)}{\xi}\right] \eta=0 \text { in which } & \\
\text { (b) } k(\lambda)=\kappa+\kappa_{0} / \lambda+\cdots+\kappa_{n} / \lambda^{n+1}, & n \geqq 0, \\
\text { (c) } \kappa=-(1 / 2) p_{1}(0) \psi^{4}(0) &
\end{array}
$$

and $\kappa_{0}, \kappa_{1}, \cdots, \kappa_{n}$ are unspecified constants.

Because equation (2.5) is second order linear, and because of the form of the transformation (2.4), it is familiar that $v(x, \lambda)$ will satisfy a second order linear differential equation. The application of $d / d x=\xi^{\prime} d / d \xi$ to $v(x, \lambda)$ yields, when (2.3) is taken into account,

$$
v^{\prime \prime}=\psi\left(\xi^{\prime}\right)^{2}\left[\frac{d^{2} \eta}{d \xi^{2}}+\frac{1}{2 \xi} \frac{d \eta}{d \xi}\right]+\psi^{\prime \prime} \eta
$$

or, with (2.5) and (2.2),

$$
v^{\prime \prime}=\left[\left(\frac{1}{4}-\frac{k}{\xi}\right)\left(\xi^{\prime}\right)^{2}+\frac{\psi^{\prime \prime}}{\psi}\right] v .
$$

From this, and from the formulas (2.1) and (2.2), it follows that $v(x, \lambda)$ satisfies the differential equation

$$
\begin{aligned}
& \text { (a) } \frac{d^{2} v}{d x^{2}}-R(x, \lambda) v=0 \text { where } \\
& \text { (b) } R(x, \lambda)=\lambda^{2} p_{0}+\lambda p_{1}(0) \frac{\psi^{4}(0)}{\psi^{4}}+\frac{\psi^{\prime \prime}}{\psi}-\frac{2}{\psi^{4}} \sum_{j=0}^{n} \frac{\kappa_{j}}{\lambda^{j}} .
\end{aligned}
$$

Equation (2.5a) is normalized by the transformation $\eta=\xi^{-1 / 4} M$ to the confluent hypergeometric equation,

$$
\frac{d^{2} M}{d \xi^{2}}-\left[\frac{1}{4}-\frac{k(\lambda)}{\xi}-\frac{1-4 m^{2}}{4 \xi^{2}}\right] M=0,
$$

in which $m$ has the value 1/4. Equation (2.7) has been the subject of considerable investigation, and its solutions are to be regarded as known. In terms of these, equation (2.6), which is seen to resemble the given equation (1.1) 
to a certain extent, is solvable, with solutions of the form

$$
v=\psi(x) \xi^{-1 / 4} M(\xi, \lambda) .
$$

By analogy with Langer [3], equation (2.6) will henceforth be designated as the "first solvable approximating equation."

Let the wronskian of a pair of functions $f(x)$ and $\tilde{f}(x)$ be designated by

$$
\text { wron }(f, \tilde{f} ; x)=\left|\begin{array}{ll}
f(x) & \tilde{f}(x) \\
\frac{d f(x)}{d x} & \frac{d \tilde{f}(x)}{d x}
\end{array}\right| .
$$

If $M(\xi)$ and $\tilde{M}(\xi)$ are a pair of solutions of the Whittaker equation, and $v(x)$ and $\tilde{v}(x)$ are the corresponding solutions of the first approximating equation, computation from the relation (2.8) and a substitution from (2.2) show that

$$
\text { wron }(v, \tilde{v} ; x)=(2 \lambda)^{1 / 2} \text { wron }(M, \tilde{M} ; \xi) .
$$

Hence $v$ and $\tilde{v}$ are independent provided $M$ and $\tilde{M}$ are. Since neither differential equation has a first derivative term, neither wronskian can depend upon the differentiation variable-both are functions of $\lambda$ alone.

3. The second approximating equation. It is now proposed to make use of the first approximating equation to obtain another differential equation, still solvable, and more closely resembling the given equation. Let $\mu_{0}(x)$ and $\mu_{1}(x)$ be defined by

$$
\begin{aligned}
& \mu_{0}=\cosh \theta \text { where } \theta(x)=\int_{0}^{x} \frac{p_{1}(s)-p_{1}(0) \psi^{4}(0) / \psi^{4}(s)}{2 \phi(s)} d s, \\
& \mu_{1}=\frac{\sinh \theta}{\phi} .
\end{aligned}
$$

The integrand of $\theta(x)$ is evidently bounded, its numerator vanishing to at least as high an order as its denominator at $s=0$. Hence $\theta(x)$ vanishes at least to the order of $x$ at the origin, so that $\mu_{1}(x)$ is only indeterminate there, and becomes an indefinitely differentiable function upon proper definition at this single point. It may be established from (3.1) that

$$
\begin{aligned}
\text { (a) } \mu_{0}^{2}-p_{0} \mu_{1}^{2} & \equiv 1, \\
\text { (b) } 2 p_{0} \mu_{1}^{\prime}+p_{0}^{\prime} \mu_{1} & =\left[p_{1}-p_{1}(0) \frac{\psi^{4}(0)}{\psi^{4}}\right] \mu_{0}, \\
\text { (c) } 2 \mu_{0}^{\prime} & =\left[p_{1}-p_{1}(0) \frac{\psi^{4}(0)}{\psi^{4}}\right] \mu_{1} .
\end{aligned}
$$

In terms of the functions $\mu_{0}(x)$ and $\mu_{1}(x)$, and of $v(x, \lambda)$, any solution of 
the first approximating equation (2.6), let $z(x, \lambda)$ be defined by the formula

$$
\text { (a) } z=\mu_{0} v+\frac{\mu_{1}}{\lambda} v^{\prime} \text {. }
$$

Differentiating, and substituting for $v^{\prime \prime}$ from (2.6),

$$
\begin{aligned}
& \text { (b) } z^{\prime}=\left(\mu_{0}^{\prime}+\frac{\mu_{1}}{\lambda} R\right) v+\left(\mu_{0}+\frac{\mu_{1}^{\prime}}{\lambda}\right) v^{\prime}, \\
& \text { (c) } z^{\prime \prime}=\left(\mu_{0}^{\prime \prime}+\mu_{0} R+\frac{2 \mu_{1}^{\prime}}{\lambda} R+\frac{\mu_{1}}{\lambda} R^{\prime}\right) v+\left(2 \mu_{0}^{\prime}+\frac{\mu_{1}^{\prime \prime}}{\lambda}+\frac{\mu_{1}}{\lambda} R\right) v^{\prime} .
\end{aligned}
$$

$v$ and $v^{\prime}$ may be eliminated from equations (3.3), the eliminant being

$$
\left|\begin{array}{ccc}
z & z^{\prime} & z^{\prime \prime} \\
\mu_{0} & \mu_{0}^{\prime}+\frac{\mu_{1}}{\lambda} R & \mu_{0}^{\prime \prime}+\mu_{0} R+\frac{2 \mu_{1}^{\prime}}{\lambda} R+\frac{\mu_{1}}{\lambda} R^{\prime} \\
\frac{\mu_{1}}{\lambda} & \mu_{0}+\frac{\mu_{1}^{\prime}}{\lambda} & 2 \mu_{0}^{\prime}+\frac{\mu_{1}^{\prime \prime}}{\lambda}+\frac{\mu_{1}}{\lambda} R
\end{array}\right|=0 .
$$

Hence $z$ satisfies the differential equation

$$
D_{0} \frac{d^{2} z}{d x^{2}}-H \frac{d z}{d x}-J z=0
$$

where

$$
\begin{aligned}
& \text { (a) } D_{0}=\left|\begin{array}{cc}
\mu_{0} & \mu_{0}^{\prime}+\frac{\mu_{1}}{\lambda} R \\
\frac{\mu_{1}}{\lambda} & \mu_{0}+\frac{\mu_{1}^{\prime}}{\lambda}
\end{array}\right|, \\
& \text { (b) } H=\left|\begin{array}{cc}
\mu_{0} & \mu_{0}^{\prime \prime}+\frac{2 \mu_{1}^{\prime}}{\lambda} R+\frac{\mu_{1}}{\lambda} R^{\prime} \\
\frac{\mu_{1}}{\lambda} & 2 \mu_{0}^{\prime}+\frac{\mu_{1}^{\prime \prime}}{\lambda}
\end{array}\right|, \\
& \text { (c) } J=\left|\begin{array}{cc}
\mu_{0}^{\prime \prime}+\mu_{0} R+\frac{2 \mu_{1}^{\prime}}{\lambda} R+\frac{\mu_{1}}{\lambda} R^{\prime} & \mu_{0}^{\prime}+\frac{\mu_{1}}{\lambda} R \\
2 \mu_{0}^{\prime}+\frac{\mu_{1}^{\prime}}{\lambda}+\frac{\mu_{1}}{\lambda} R & \mu_{0}+\frac{\mu_{1}^{\prime}}{\lambda}
\end{array}\right| .
\end{aligned}
$$

No use has been made thus far of the specific relations (3.2) satisfied by 
$\mu_{0}$ and $\mu_{1}$. The first of these, (3.2a), is now applied to the determinant $D_{0}$ to obtain the expansion:

$$
D_{0}=1+\frac{1}{\lambda}\left[\mu_{0} \mu_{1}^{\prime}-\mu_{0}^{\prime} \mu_{1}-\mu_{1}^{2} p_{1}(0) \frac{\psi^{4}(0)}{\psi^{4}}\right]-\frac{\mu_{1}^{2}}{\lambda^{2}}\left[\frac{\psi^{\prime \prime}}{\psi}-\frac{2}{\psi^{4}} \sum_{j=0}^{n} \frac{\kappa_{j}}{\lambda^{i}}\right]
$$

from which it is deduced that both $D_{0}$ and its reciprocal are bounded from zero when $|\lambda|>N$. Since, as may be verified directly, $H=D_{0}^{\prime}$, equation (3.4) is normalized by the transformation

$$
z=z D_{0}^{-1 / 2}
$$

to the equation

$$
\begin{aligned}
& \text { (a) } \frac{d^{2} z}{d x^{2}}-S(x, \lambda)_{z}=0 \text { in which } \\
& \text { (b) } S=\frac{J}{D_{0}}+\frac{3}{4}\left[\frac{D_{0}^{\prime}}{D_{0}}\right]^{2}-\frac{1}{2} \frac{D_{0}^{\prime \prime}}{D_{0}} .
\end{aligned}
$$

The only term on the right of (3.8b) which becomes infinite with $\lambda$ is $J / D_{0}$. Inspection of the determinants (3.5) shows that the indicated division can be carried out in part to obtain

$$
\begin{aligned}
& \text { (a) } \frac{J}{D_{0}}=R+\frac{J_{0}}{D_{0}} \text { with } \\
& \text { (b) } J_{0}=\left|\begin{array}{cc}
\mu_{0}^{\prime \prime}+\frac{2 \mu_{1}^{\prime}}{\lambda} R+\frac{\mu_{1}}{\lambda} R^{\prime} & \mu_{0}^{\prime}+\frac{\mu_{1}}{\lambda} R \\
2 \mu_{0}^{\prime}+\frac{\mu_{1}^{\prime \prime}}{\lambda} & \mu_{0}+\frac{\mu_{1}^{\prime}}{\lambda}
\end{array}\right|
\end{aligned}
$$

It is seen that, except for the term $R$ written explicitly in (3.9a), the functions $R(x, \lambda)$ and $R^{\prime}(x, \lambda)$ come into the expression for $S(x, \lambda)$ invariably multiplied by quantities of the order of $1 / \lambda$ or smaller. Consequently, the only term of the order of $\lambda$ is, from (2.6), $\lambda^{2} p_{0}(x)$. Since by formula (3.6), $D_{0}$ is bounded with leading term 1 , the coefficient of the term of order $\lambda$ in $S(x, \lambda)$ is

$$
p_{1}(0) \frac{\psi^{4}(0)}{\psi^{4}}+\left|\begin{array}{cc}
2 \mu_{1}^{\prime} p_{0}+\mu_{1} p_{0}^{\prime} & \mu_{1} p_{0} \\
2 \mu_{0}^{\prime} & \mu_{0}
\end{array}\right| .
$$

Because $\mu_{0}$ and $\mu_{1}$ satisfy the relations (3.2), this simplifies to $p_{1}(x)$. Consequently, the coefficient $S(x, \lambda)$ of equation (3.8) takes the form

(a) $S(x, \lambda)=\lambda^{2} p_{0}(x)+\lambda p_{1}(x)+T(x, \lambda)$

where, because $D_{0}^{-1}$ can be expanded in descending powers of $\lambda, T(x, \lambda)$ is of 
the form

$$
\text { (b) } T(x, \lambda)=\sum_{j=0}^{\infty} t_{j}(x) / \lambda^{i} \text {. }
$$

The constant $\kappa_{m}$, with $m=0,1, \cdots$, or $n$, comes into the expression for $S(x, \lambda)$ wherever $R(x, \lambda)$ or $R^{\prime}(x, \lambda)$ does. As remarked above, except for the term $R$ written explicitly in (3.9a), these functions are always multiplied by quantities of the order of $1 / \lambda$ or smaller. Consequently, $\kappa_{m}$ occurs linearly in $t_{m}(x)$ (multiplied by $-2 / \psi^{4}$ ), but is absent from every $t_{j}(x)$ with $j<m$.

Equation (3.8), which has the explicit solution

$$
z=D_{0}^{-1 / 2}\left[\mu_{0} v+\frac{\mu_{1}}{\lambda} v^{\prime}\right],
$$

is to be referred to as the "second solvable approximating equation." If $v$ and $\tilde{v}$ are a pair of solutions of the first approximating equation, and $z$ and $\bar{z}$ are the corresponding functions defined by (3.3a), then from (3.3) and (3.5a)

$$
\operatorname{wron}(z, \tilde{z} ; x)=\left|\begin{array}{cc}
\mu_{0} & \frac{\mu_{1}}{\lambda} \\
\mu_{0}^{\prime}+\frac{\mu_{1}}{\lambda} R & \mu_{0}+\frac{\mu_{1}^{\prime}}{\lambda}
\end{array}\right| \cdot\left|\begin{array}{cc}
v & \tilde{v} \\
v^{\prime} & \tilde{v}^{\prime}
\end{array}\right|=D_{0} \text { wron }(v, v ; x) .
$$

For the corresponding $z$ and $\bar{z}$ defined by (3.7),

$$
\text { wron }(\bar{z}, \bar{z}, x)=D_{0}^{-1} \cdot \text { wron }(z, \tilde{z} ; x)
$$

and so

$$
\text { wron }(z, \bar{z}, x)=\text { wron }(v, \tilde{v} ; x) \text {. }
$$

Hence $z$ and $\bar{z}$ are independent provided $v$ and $\bar{v}$ are.

4. The related equation. A solvable differential equation is now to be obtained which formally approximates the given equation to any desired degree of accuracy. Let $\alpha_{0}(x), \tau_{0}(x)$, and $\beta_{0}(x)$ be defined in terms of (1.2) and (3.10) by

$$
\begin{array}{ll}
\text { (a) } \alpha_{0} \equiv 1, \quad \text { (b) } \tau_{0}=\left(q_{0}-t_{0}\right) \text {, }
\end{array}
$$

(c) $\beta_{0}=\frac{1}{\phi} \int_{0}^{x} \frac{\tau_{0}(s)}{2 \phi(s)} d s$.

$\kappa_{0}$, which occurs linearly in $t_{0}(x)$, may be chosen so that $\tau_{0}(x)$ has a zero at the origin, and this choice is to be made. As a result, the integrand of $\beta_{0}$ is bounded near the origin, and $\beta_{0}$ itself is only indeterminate there. Suitably defined at 
this single point, $\beta_{0}(x)$ is indefinitely differentiable on $(a, b)$. It is to be noted that quantities (4.1) do not involve any $\kappa_{m}$ other than $\kappa_{0}$.

Let $\alpha_{1}(x), \tau_{1}(x)$, and $\beta_{1}(x)$ be defined by
(a) $\alpha_{1} \equiv 0$,
(b) $\tau_{1}=\left(q_{1}-t_{1}\right)-2 p_{1} \beta_{0}^{\prime}-p_{1}^{\prime} \beta_{0}$,
(c) $\beta_{1}=\frac{1}{\phi} \int_{0}^{x} \frac{\tau_{1}(s)}{2 \phi(s)} d s$.

$\kappa_{1}$, which occurs linearly in $t_{1}(x)$, is now chosen so that $\tau_{1}(x)$ has a zero at the origin. Hence $\beta_{1}(x)$ is a function which, by suitable definition at the origin, is indefinitely differentiable on $(a, b)$. Evidently the quantities (4.2) do not involve any $\kappa_{m}$ other than $\kappa_{0}$ and $\kappa_{1}$.

In this fashion a sequence of functions is to be defined. At the $\nu$ th stage, and in terms of previously defined quantities, let $\alpha_{\nu}(x), \beta_{\nu}(x)$, and $\tau_{\nu}(x)$ be defined by

$$
\begin{aligned}
& \text { (a) } \alpha_{\nu}=\frac{1}{2} \int_{0}^{x}\left[\beta_{\nu-2}^{\prime \prime}+\sum_{j=0}^{\nu-2}\left(q_{\nu-j-2}-t_{\nu-j-2}\right) \beta_{j}\right] d t \text {, } \\
& \text { (b) } \tau_{\nu}=\sum_{j=0}^{\nu}\left(q_{\nu-j}-t_{\nu-j}\right) \alpha_{j}-\left(2 p_{1} \beta_{\nu-1}^{\prime}+p_{1}^{\prime} \beta_{\nu-1}\right) \\
& -\sum_{j=0}^{\nu-2}\left(2 t_{\nu-j-2} \beta_{j}^{\prime}+t_{\nu-j-2} \beta_{j}\right)-\alpha_{\nu}^{\prime \prime}, \\
& \text { (c) } \beta_{\nu}=\frac{1}{\phi} \int_{0}^{x} \frac{\tau_{\nu}(s)}{2 \phi(s)} d s \text {. }
\end{aligned}
$$

$\kappa_{\nu}$, which occurs linearly in $t_{\nu}(x)$, is chosen so that $\tau_{\nu}$ has a zero at $x=0$. $\beta_{\nu}(x)$, with definition suitably extended to the origin, is therefore indefinitely differentiable on $(a, b)$. The quantities (4.2) involve $\kappa_{0}, \kappa_{1}, \cdots, \kappa_{\nu}$ only.

The sequence is to be terminated with the stage $\nu=n$. ( $n$, which was introduced in (2.5), is arbitrary.) At this stage all of the $\kappa_{0}, \kappa_{1}, \cdots, \kappa_{n}$ will be determined.

In terms of the above functions define

$$
\begin{aligned}
& \text { (a) } A(x, \lambda)=\sum_{j=0}^{n} \alpha_{j}(x) / \lambda^{j} \\
& \text { (b) } B(x, \lambda)=\sum_{j=0}^{n} \beta_{j}(x) / \lambda^{j}
\end{aligned}
$$

$$
\text { (c) } D_{1}(x, \lambda)=\left|\begin{array}{cc}
A & A^{\prime}+\frac{B}{\lambda^{2}} S \\
\frac{B}{\lambda^{2}} & A+\frac{B^{\prime}}{\lambda^{2}}
\end{array}\right| \text {. }
$$


Because $\alpha_{0} \equiv 1$ and $\alpha_{1} \equiv 0$, therefore $A^{2}$, and hence $D_{1}$, differs from unity by a quantity of order $1 / \lambda^{2}$. Thus both $D_{1}$ and its reciprocal are bounded from zero when $|\lambda|>N$.

Consider now the function $y(x, \lambda)$ :

$$
y=D_{1}^{-1 / 2}\left[A z+B z^{\prime} / \lambda^{2}\right] .
$$

By reasoning similar to that of $\$ 3$ it may be shown $\left({ }^{4}\right)$ that $y$ satisfies the differential equation

$$
\frac{d^{2} y}{d x^{2}}-\left[\lambda^{2} p_{0}+\lambda p_{1}+Q(x, \lambda)-\frac{\Omega(x, \lambda)}{\lambda^{n+1}}\right] y=0
$$

in which $\Omega(x, \lambda)$ is a function which is continuous and is bounded when $|\lambda|>N$. This equation, which resembles the given equation to terms in $1 / \lambda^{n}$, will be referred to as the "related equation." Its explicitly known solutions are given by (4.5).

A solution $y(x, \lambda)$ of the related equation may be written directly in terms of the corresponding solution of the first approximating equation by combining the expressions (4.5) and (3.11), eliminating $v^{\prime \prime}$ with (2.6). The expression is

$$
\begin{aligned}
& \text { (a) } y(x, \lambda)=E_{0}(x, \lambda) v(x, \lambda)+E_{1}(x, \lambda) v^{\prime}(x, \lambda) / \lambda \text { with } \\
& \text { (b) } \quad E_{0}=\left(D_{0} D_{1}\right)^{-1 / 2}\left[A \mu_{0}+\frac{B}{\lambda}\left(\frac{\mu_{1}}{\lambda^{2}} R+\frac{\mu_{0}^{\prime}}{\lambda}-\frac{\mu_{0}}{2 \lambda} \frac{D_{0}^{\prime}}{D_{0}}\right)\right] \text {, and } \\
& \text { (c) } E_{1}=\left(D_{0} D_{1}\right)^{-1 / 2}\left[A \mu_{1}+\frac{B}{\lambda}\left(\mu_{0}+\frac{\mu_{1}^{\prime}}{\lambda}-\frac{\mu_{1}}{2 \lambda} \frac{D_{0}^{\prime}}{D_{0}}\right)\right] .
\end{aligned}
$$

If $z$ and $\bar{z}$ are a pair of solutions of the second approximating equation, then an argument similar to one employed in $\$ 3$ shows that for the corresponding pair of solutions, $y$ and $\tilde{y}$, of the related equation,

$$
\text { wron }(y, \tilde{y} ; x)=\text { wron }(z, \bar{z} ; x) \text {. }
$$

The result is now immediate from (3.12) that

$$
\text { wron }(y, \tilde{y} ; x)=\text { wron }(v, \tilde{v}, x) \text {. }
$$

The behavior of the functions $y$ may be deduced by (4.7) from that of the $v$ 's or, what comes to the same thing, from the behavior of the corresponding pair of solutions of the Whittaker equation (2.7).

5. The solutions of the related equation. An independent pair of solutions of the Whittaker equation, those generally designated by $M_{k, m}(\xi)$, are given for all finite values of the variable $\xi$ and the parameter $\lambda$ by ${ }^{(5)}$

(4) The method is formally identical with that of $[3,86]$.

(5) Notation and results quoted in this section are taken from [5, Chap. 16]. The equations (5.1) and (5.2) are found on p. 332. 


$$
M_{k, m}(\xi)=\xi^{1 / 2+m} e^{-\xi / 2}\left[1+\frac{1 / 2+m-k}{2 m+1} \xi+\cdots\right], m= \pm 1 / 4,
$$

the series being uniformly convergent on a bounded range of $\xi$ and $k . M_{k, m}(\xi)$ is a single-valued function of arg $\xi$ by the convention of $\S 2$, and satisfies the known relations

$$
M_{k, m}(\xi)=i^{\nu} \cdot e^{m \nu \pi i} M_{\sigma k, m}\left(\xi e^{-\nu \pi i}\right)
$$

where $\nu$ is any integer and where

$$
\sigma=(-1)^{p} .
$$

The functions $v_{ \pm 1 / 4}(x, \lambda)$, defined in terms of $M_{k, \pm 1 / 4}(\xi)$ by the formula

$$
v_{m}(x, \lambda)=\psi(x) \xi^{-1 / 4} M_{k(\lambda), m}(\xi), \quad m= \pm 1 / 4,
$$

satisfy the first approximating equation throughout the interval $(a, b)$. Over a domain of values $(x, \lambda)$ restricted in such a way that $|\lambda|>N$ but $\xi(x, \lambda)$ remains bounded, $k(\lambda)$ is confined to a neighborhood of the point $\kappa$, and consequently the functions $v_{m}(x, \lambda)$ and $v_{m}^{\prime}(x, \lambda) / \lambda^{1 / 2}$ are bounded uniformly. The corresponding solutions of the related equation, defined by formula

$$
y_{m}(x, \lambda)=E_{0}(x, \lambda) v_{m}(x, \lambda)+E_{1}(x, \lambda) v_{m}^{\prime}(x, \lambda) / \lambda, \quad m= \pm 1 / 4,
$$

are also uniformly bounded on the restricted domain. From (2.10), (4.8), and (5.1)

$$
\text { wron }\left[y_{1 / 4}, y_{-1 / 4} ; x\right]=-\left(\frac{\lambda}{2}\right)^{1 / 2} \text {. }
$$

When $\xi(x, \lambda)$ is unbounded it becomes appropriate to introduce the Whittaker functions which are designated by $W_{\sigma k, 1 / 4}\left(\xi e^{-\nu \pi i}\right)$, where $\nu$ is any integer, and $\sigma$ is defined by (5.3). For brevity, let

$$
W_{\sigma k, 1 / 4}\left(\xi e^{-\nu \pi i}\right)=W_{\nu}(\xi) .
$$

$W_{k, 1 / 4}(\xi)$ is defined in terms of the functions $M_{k, m}(\xi)$ by the formula $\left({ }^{6}\right)$

$$
W_{k, 1 / 4}(\xi)=\frac{\Gamma(-1 / 2)}{\Gamma(1 / 4-k)} M_{k, 1 / 4}(\xi)+\frac{\Gamma(1 / 2)}{\Gamma(3 / 4-k)} M_{k,-1 / 4}(\xi) .
$$

Consequently, by (5.2),

$$
W_{\nu}(\xi)=\frac{e^{-(1 / 4) \nu \pi i} \Gamma(-1 / 2)}{i^{\nu} \Gamma(1 / 4-\sigma k)} M_{k, 1 / 4}(\xi)+\frac{e^{(1 / 4) \nu \pi i} \Gamma(1 / 2)}{i^{\nu} \Gamma(3 / 4-\sigma k)} M_{k,-1 / 4}(\xi),
$$

which shows incidentally that $W_{\nu}(\xi)$ is a solution of the differential equation (2.7). When $\xi$ is large and in the sector

(e) $[5$, p. 340$]$. 


$$
\Sigma_{\nu}(\xi): \quad(\nu-3 / 2) \pi+\epsilon \leqq \arg \xi \leqq(\nu+3 / 2) \pi-\epsilon,
$$

with $\epsilon$ a small fixed positive number, then $W_{\nu}$ is expressible by the asymptotic formula $\left({ }^{7}\right)$

$$
W_{\nu}(\xi)=e^{-(1 / 2) \sigma \xi}\left(\xi e^{-\nu \pi i}\right) \sigma k\left[1+\frac{O(1)}{\xi}\right] .
$$

The function designated here by $O(1)$, as well as its $\xi$ derivative, may be shown to be uniformly bounded when $|\xi|>N$ and $|k|<M\left(^{8}\right)$.

The sector $S_{\nu}(\xi)$, defined by$$
\text { (a) } \quad(\nu-1 / 2) \pi \leqq \arg \xi \leqq(\nu+1 / 2) \pi
$$$$
\text { if } \Re[\sigma k] \leqq 0
$$$$
\text { (b) }(\nu-1 / 2) \pi+\epsilon \leqq \arg \xi \leqq(\nu+1 / 2) \pi-\epsilon \quad \text { if } \Re[\sigma k]>0 \text {, }
$$

is overlapped considerably by each of the sectors $\Sigma_{\nu-1}(\xi)$ and $\Sigma_{\nu+1}(\xi)$ and in case (b) is covered completely by either of these.

Consider now the pair of functions $W_{\nu}(\xi)$ and $W_{\nu+1}(\xi)$. When $|\xi|>N$ and $\arg \xi$ is on the range

$$
(\nu-1 / 2) \pi+\epsilon \leqq \arg \xi \leqq(\nu+3 / 2) \pi-\epsilon,
$$

then both functions may be expressed asymptotically by means of (5.10). Their wronskian, being independent of $\xi$, may be evaluated from these formulas in the limit as $\xi$ becomes infinite, and has the value

$$
\text { wron }\left[W_{\nu}, W_{\nu+1} ; \xi\right]=\sigma e^{\sigma k \pi i} \text {. }
$$

Hence $W_{\nu}(\xi)$ and $W_{\nu+1}(\xi)$ are independent.

On the sector $S_{\nu}(\xi)$, the function $e^{-(1 / 2) \sigma \xi \xi \sigma k}$ is bounded. Hence any constant linear combination

$$
C_{1} W_{\nu}(\xi)+C_{2} W_{\nu+1}(\xi) \text {, with } C_{2} \neq 0,
$$

may be represented asymptotically there by

$$
C_{2} e^{(1 / 2) \sigma \xi}\left(\xi e^{-(\nu+1) \pi i}\right)^{-\sigma k} O(1),
$$

the bounded term $C_{1} W_{\nu}(\xi)$ having been incorporated into the function $O(1)$. Because this can be done, $W_{\nu+1}(\xi)$ is said to be dominant over $W_{\nu}(\xi)$ on this sector; on the sector $S_{v+1}(\xi)$, the roles of dominant and subdominant function are reversed.

On the (sometimes smaller) sector

$$
(\nu-1 / 2) \pi+\epsilon \leqq \arg \xi \leqq(\nu+1 / 2) \pi-\epsilon
$$

the dominance is even stronger, for here $e^{-(1 / 2) \sigma \xi \xi \sigma k}$ is of infinitely small

(7) $[5$, pp. 337, 339].

(8) In general $O(1)$ will designate a function which, on a configuration of values of the parameter and variables, is bounded and is continuous in the variables. 
order, i.e. is $O\left(\xi^{-n}\right)$ for every integer $n$, so that the asymptotic expansion of the above linear combination is identical in all terms with that of $C_{2} W_{\nu+1}(\xi)$. Hence only the pure subdominant solution may be distinguished by its asymptotic form; all other solutions are asymptotically identical $\left({ }^{9}\right)$.

Outside of the range of arg $\xi$ for which the forms (5.10) are valid, these may be used in conjunction with the relations

$$
\begin{aligned}
W_{k, 1 / 4}\left(\xi e^{\nu \pi i}\right)= & \frac{2 \pi i^{\nu-1} e^{-k \pi i} \sin (1 / 4) \pi \nu}{\Gamma(1 / 4-k) \Gamma(3 / 4-k)} W_{-k, 1 / 4}\left(\xi e^{-\pi i}\right) \\
& +i^{\nu}\left[\sin (1 / 4) \pi(\nu+2)+e^{-2 k \pi i} \sin (1 / 4) \pi \nu\right] W_{k, 1 / 4}(\xi),
\end{aligned}
$$

valid when $\nu$ is an even integer, and

$$
\begin{gathered}
W_{k, 1 / 4}\left(\xi e^{\nu \pi i}\right)=i^{\gamma-1}\left[e^{2 k \pi i} \sin (1 / 4) \pi(\nu+1)+\sin (1 / 4) \pi(\nu-1)\right] W_{k, 1 / 4}\left(\xi e^{-\pi i}\right) \\
+\frac{2 \pi i^{\nu} e^{k \pi i} \sin (1 / 4) \pi(\nu+1)}{\Gamma(1 / 4-k) \Gamma(3 / 4-k)} W_{-k, 1 / 4}(\xi),
\end{gathered}
$$

where $\nu$ is odd.

The relations (5.13) are a corollary of their analogues (5.2), following, after some computation, by the definition (5.8).

Formula (5.13a) may be applied in particular to show that (5.14a)

$$
W_{\nu+2}(\xi)=\frac{-2 \pi i e^{-\sigma k \pi i}}{\Gamma(1 / 4-\sigma k) \Gamma(3 / 4-\sigma k)} W_{\nu+1}(\xi)-e^{-2 \sigma k \pi i} W_{\nu}(\xi) .
$$

Consequently, $W_{\nu+2}(\xi)$ and $W_{\nu}(\xi)$ are linearly independent provided that neither of the $\Gamma$-functions is infinite, thus certainly when neither of $k \pm 1 / 4$ is an integer. It is immediately evident from (5.1) and (5.8) that there are only four essentially different functions $W_{\nu}(\xi)$, and what has just been shown is that any two of these are linearly independent, although the above mentioned exception can occur. It follows from $(5.13 \mathrm{~b})$ that

$$
W_{\nu+2}(\xi)=\frac{-2 \pi i e^{\sigma k \pi i}}{\Gamma(1 / 4-\sigma k) \Gamma(3 / 4-\sigma k)} W_{\nu-1}(\xi)-e^{2 \sigma k \pi i} W_{\nu}(\xi) .
$$

Either of the formulas (5.14) may be applied to obtain an asymptotic expression for $W_{\nu+2}(\xi)$ on at least a portion of the sector $S_{\nu}(\xi)$, and the results may be combined to yield an expression valid over this entire sector. Hence, when $|\xi|>M$ and on the sector $S_{\nu}(\xi)$,

$$
W_{\nu+2}(\xi)=\frac{-2 \pi i e^{\sigma k \pi i \nu}}{\Gamma(1 / 4-\sigma k) \Gamma(3 / 4-\sigma k)} e^{(1 / 2) \sigma \xi \xi-\sigma k}\left[1+\frac{O(1)}{\xi}\right] .
$$

(9) Except of course for the constant factor. It should be pointed out that this factor may depend upon the parameter $\lambda$, and that the character of a solution as dominant or subdominant may not be uniform over a region of $\lambda$, e.g. if $C(\lambda)$ has zeroes there. 
The formulas

$$
\begin{aligned}
\text { (a) } v_{\nu}(x, \lambda) & =\psi(x) \xi^{-1 / 4} W_{\nu}(\xi, \lambda), \\
\text { (b) } y_{\nu}(x, \lambda) & =E_{0}(x, \lambda) v_{\nu}(x, \lambda)+E_{1}(x, \lambda) v_{\nu}^{\prime}(x, \lambda) / \lambda, \\
\nu & =0,1,2,3(\bmod 4),
\end{aligned}
$$

define solutions of the first approximating equation and related equation respectively which are alternative to those given in (5.4) and (5.5). From the behavior of the latter for small $\xi$, together with the equation $(5.8)\left({ }^{10}\right)$,

(a) $y_{v}(x, \lambda)=O(1)$,

(b) $y_{\nu}^{\prime}(x, y)=O\left(\lambda^{1 / 2}\right)$,

$|\xi| \leqq M,|\lambda|>N$

When $|\xi| \geqq M$ and in the sector $\Sigma_{\nu}(\xi)$

$$
\begin{aligned}
& \text { (a) } y_{\nu}(x, \lambda)=e^{-(1 / 2) \sigma \xi \xi \sigma k-1 / 4} O(1), \\
& \text { (b) } y_{\nu}^{\prime}(x, \lambda)=e^{-(1 / 2) \sigma \xi \xi \sigma k-1 / 4} O(\lambda),
\end{aligned}
$$

these formulas following from (5.10) and (4.7).

A pair of the functions $y$ will be called adjacent if their subscripts differ by 1 or 3 , modulo 4 , or opposite if their subscripts differ by 2, modulo 4 . An adjacent pair $y_{v}(x, \lambda)$ and $y_{v+1}(x, \lambda)$ are linearly independent, with

$$
\text { wron }\left[y_{v}, y_{v+1} ; x\right]=\sigma(2 \lambda)^{1 / 2} e^{\sigma k \pi i}
$$

while an opposite pair are independent for $|\lambda|>N$ provided neither $\kappa \pm 1 / 4$ is an integer.

6. Representation of solutions of the given equation. If $\hat{y}(x), \tilde{y}(x)$ and $y(x)$ are solutions of the related equation, the first two being linearly independent, and $x_{0}$ is any point of the interval $(a, b)$, then the function $u(x)$ which is defined by the integral equation

$$
u(x)=y(x)+\int_{x_{0}}^{x} \frac{\hat{y}(x) \tilde{y}(t)-\tilde{y}(x) \hat{y}(t)}{\text { wron }[y, \tilde{y} ; t]} \frac{\Omega(t, \lambda)}{\lambda^{n+1}} u(t) d t
$$

is a solution of the given differential equation. This result, which is obtainable from classical theory (by considering the given equation as a nonhomogeneous generalization of the related equation), may be verified directly. Differentiating (6.1) leads to the following expression for $u^{\prime}(x)$ :

$$
u^{\prime}(x)=y^{\prime}(x)+\int_{x_{0}}^{x} \frac{\hat{y}^{\prime}(x) \tilde{y}(t)-\tilde{y}^{\prime}(x) \hat{y}(t)}{\text { wron }[\hat{y}, \tilde{y}, t]} \frac{\Omega(t, \lambda)}{\lambda^{n+1}} u(t) d t .
$$

The kernel of (6.1) does not depend upon the particular choice made of a pair of solutions $y(x)$ and $\tilde{y}(x)$. Consequently (6.1) establishes a unique correspondence between a solution $y(x)$ of the related equation and a solution

(10) $O\left(\lambda^{n}\right)$ means $O(1) \cdot \lambda^{n}$. 
$u(x)$ of the given equation, characterized by the evident fact that these functions, with their derivatives, are identical at the point $x_{0}$ :

$$
u\left(x_{0}\right)=y\left(x_{0}\right) ; \quad u^{\prime}\left(x_{0}\right)=y^{\prime}\left(x_{0}\right) .
$$

It is to be investigated under what circumstances the deviation of $u$ from $y$ remains small for a range or values of $x$ and $\lambda$.

Consider first the configuration of values $(x, \lambda)$ for which $|\lambda|>N$ but $\xi(x, \lambda)$ is bounded. Choose $M$, a large fixed positive number. For any fixed $\lambda$, $|\xi(x, \lambda)|$ increases monotonically with $|x|$ on either interval $(a, 0)$ or $(0, b)$. Consequently $|\xi|<M$ on a subinterval $\left(x_{-}, x_{+}\right)$of $(a, b)$ which contains the origin and whose end points vary with $\lambda$, while $|\xi|>M$ outside of $\left(x_{-}, x_{+}\right)$. With $y_{\nu}(x)$ any one of the functions introduced in the preceding section, and $x_{0}$ taken to be the origin, define $u_{\nu, 0}(x)$ by the correspondence (6.3). Hence (6.3a) $\quad u_{\nu, 0}(0, \lambda)=y_{\nu}(0, \lambda) ; \quad u_{\nu, 0}^{\prime}(0, \lambda)=y_{\nu}^{\prime}(0, \lambda), \quad \nu=0,1,2,3(\bmod 4)$.

As a convenient pair of functions $y$ and $\tilde{y}$ choose $y_{1 / 4}(x, \lambda)$ and $y_{-1 / 4}(x, \lambda)$ (defined in equation (5.5)). The following lemma( $\left.{ }^{11}\right)$ applies:

Lemma (First statement). If, for a range of value of $\lambda$ for which $|\lambda|>N$, an interval $a_{1}(\lambda) \leqq x \leqq a_{2}(\lambda)$ is defined and if, for $x, t$, and $x_{1}(\lambda)$ on this interval, the functions $f(x, \lambda)$ and $K(x, t, \lambda)$ are continuous in the variables $x$ and $t$ and such that

$$
\begin{aligned}
f(x, \lambda) & =O(1), \\
\text { (b) } \int_{x_{1}}^{x}|K(x, t, \lambda)| d t & =O\left(1 / \lambda^{j}\right), \quad i \geqq 0,
\end{aligned}
$$

then the solution $U(x, \lambda)$ of the integral equation

$$
U(x, \lambda)=f(x, \lambda)+\int_{x_{1}}^{x} K(x, t, \lambda) U(t, \lambda) d t
$$

differs from $f(x, \lambda)$ by an amount of the order of $1 / \lambda^{j}$, that is,

$$
U(x, \lambda)=f(x, \lambda)+O\left(1 / \lambda^{i}\right) .
$$

On the configuration of values $(x, \lambda)$ in question, the function $y=y_{\nu}(x)$ in the integral equation (6.1) is both continuous and bounded. In estimating the integral corresponding to $(6.4 \mathrm{~b})$ it is convenient to transform the integration variable by means of $(2.1 \mathrm{~b})$ and $(2.2)$ :

$$
d x=\psi^{2} d \xi /(2 \lambda)^{1 / 2} \xi^{1 / 2} .
$$

Since the wronskian in the integral is equal to $(\lambda / 2)^{1 / 2}$, while $y_{ \pm 1 / 4}(x)$ is bounded, therefore

(11) Proved in [3]. 


$$
\int_{0}^{x}|K(x, t, \lambda)| d t=O\left(1 / \lambda^{n+2}\right) \int_{0}^{\xi} d \xi / \xi^{1 / 2}=O\left(1 / \lambda^{n+2}\right) .
$$

Consequently, from the lemma,

(a) $u_{\nu, 0}(x, \lambda)=y_{v}(x, \lambda)+O\left(1 / \lambda^{n+2}\right)$, when $|\xi|<M$.

$u_{\nu, 0}^{\prime}(x)$ may now be computed from (6.2). Since $y_{ \pm 1 / 4}^{\prime}=O\left(\lambda^{1 / 2}\right)$,

(b) $\mu_{\nu, 0}^{\prime}(x, \lambda)=y_{\nu}^{\prime}(x, \lambda)+O\left(1 / \lambda^{n+8 / 2}\right)$, when $|\xi|<M$.

7. The solutions for unbounded $\xi$. The lemma of the previous section may be restated as follows:

LEMMA (Second statement). If, for a range of values of $\lambda$ for which $|\lambda|>N$, an interval $a_{1}(\lambda) \leqq x \leqq b_{1}(\lambda)$ is defined, and if, for $x, t$, and $x_{1}(\lambda)$ on the interval, the functions $f(x, \lambda)$ and $K(x, t, \lambda)$ are continuous in the variables $x$ and $t$ and such that

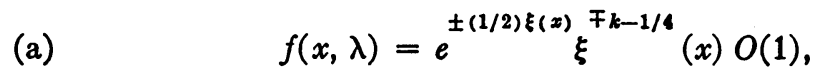

$$
\text { (b) } \int_{x_{1}}^{x}\left|e^{\mp(1 / 2)[\xi(x)-\xi(t)]}\left[\frac{\xi(x)}{\xi(t)}\right]^{ \pm k+1 / 4} K(x, t, \lambda)\right| d t=O(1 / \lambda \eta,
$$

then the solution $U(x, \lambda)$ of (6.5) is of the form

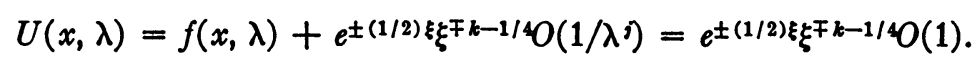

It is seen that the lemma in its original form applies directly to the integral equation for $U e^{\mp(1 / 2) \xi} \xi^{ \pm k+1 / 4}$, which is obtained by multiplying (6.5) through by $e^{\mp(1 / 2) \xi(x)} \xi^{ \pm k+1 / 4}(x)$ and rewriting the integrand.

Let the solution, $u_{r, x_{0}}(x, \lambda)$, of the given equation be defined by the initial conditions

$$
u_{v, x_{0}}\left(x_{0}, \lambda\right)=y_{v}\left(x_{0}, \lambda\right) ; \quad u_{v, \grave{x}_{0}}^{\prime}\left(x_{0}, \lambda\right)=y_{v}^{\prime}\left(x_{0}, \lambda\right) .
$$

$u_{v, x_{0}}$ is thus represented by the integral equation (6.1) when $y=y_{v}$ in that equation. The second form of the lemma is to be applied to the investigation of certain solutions, $u_{v, x_{0}}$, when $x$ is no longer restricted to the interval $\left(x_{-}, x_{+}\right)$ (whose length is of the order of $\left.1 / \lambda^{1 / 2}\right)$ but varies over the whole of $(a, b)$. The discussion applies when $|\lambda|>N, \lambda$ lies in certain fixed sectors, and $x$ is confined (for the time being) to a particular one of the subintervals $(a, 0)$ or $(0, b)$.

A typical configuration, namely

or

(a) $x>0 ; \quad-\pi / 2+\epsilon \leqq \arg \lambda \leqq \pi / 2$

if $\Re(\kappa) \leqq 0$

(b) $x>0 ; \quad-\pi / 2+\epsilon \leqq \arg \lambda \leqq \pi / 2-\epsilon$ if $\Re(\kappa)>0$ 
is chosen for detailed consideration; the discussion is then seen to carry over with little change to the general case.

I. The solution subdominant on the configuration (7.4). Consider that solution of the given differential equation which is designated by $u_{0, b}(x, \lambda)$ and which accordingly is represented by the integral equation (6.1) when $y=y_{0}$ and $x_{0}=b$ in that equation. The roles of $y$ and $\tilde{y}$ in the integrand are to be filled by $y_{0}$ and $y_{1}$ respectively.

The equation (6.1) is to be examined, first, when $x$ is on the range $x_{+} \leqq x \leqq b$. For this configuration $|\xi|>M$ and $\arg \xi=\arg \lambda$, so that the functions $y_{0}$ and $y_{1}$ are of the form

$$
\begin{aligned}
& y_{0}(x, \lambda)=e^{-(1 / 2) \xi \xi k-1 / 4} O(1), \\
& y_{1}(x, \lambda)=e^{(1 / 2) \xi \xi^{-k-1 / 4}} O(1),
\end{aligned}
$$

the first of these being subdominant. Evidently the function $y=y_{0}$ in (6.1) satisfies the condition (7.1a) of the lemma, with the lower sign holding. In the integral corresponding to $(7.1 \mathrm{~b})$ the variable $t$, as well as $x$, is on the range $\left(x_{+}, b\right)$. Consequently, if $\Delta(y, \tilde{y})$ abbreviates $y(x) \cdot \tilde{y}(t)-\hat{y}(x) \cdot \tilde{y}(t)$, then in the integrand

$$
\begin{aligned}
e^{[\xi(x)-\xi(t)] / 2}\left[\frac{\xi(x)}{\xi(t)}\right]^{-k+1 / 4} \Delta\left(y_{0}, y_{1}\right) & =\xi^{-1 / 2}(t)\left[O(1)+e^{\xi(x)-\xi(t)}\left[\frac{\xi(x)}{\xi(t)}\right]^{-2 k} O(1)\right] \\
& =\xi^{-1 / 2}(t) O(1) .
\end{aligned}
$$

The second equality requires justification: Since $t>x$, arg $[\xi(t)-\xi(x)]$ $=\arg \lambda$ and therefore $e^{\xi(x)-\xi(t)}$ is bounded. However when $\Re(\kappa)>0$ the quantity $[\xi(x) / \xi(t)]^{-2 k}$ is unbounded for small values of the bracketed ratio. Suppose that $\xi(x) / \xi(t)=\delta$, a small positive number. Then $\min |\xi(t)-\xi(x)|$ $=M(1 / \delta-1)$, the value being assumed when $x=x_{+}$, and $\min \Re(\arg \lambda)$

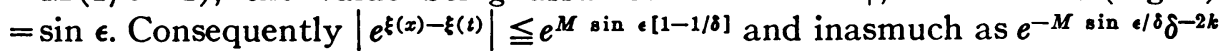
is bounded for small $\delta$, the proof is completed. If now the integration variable is transformed by (6.7) and the wronskian evaluated by (5.16), the integral in question is seen to be of the form

$$
O\left(1 / \lambda^{n+2}\right) \int_{\xi(x)}^{\xi(b)} d \xi / \xi=O\left(1 / \lambda^{n+1}\right)
$$

Accordingly the conclusion may be drawn from the lemma that

(a) $u_{0, b}(x, \lambda)=y_{0}(x, \lambda)+e^{-(1 / 2) \xi \xi k-1 / 4} O\left(1 / \lambda^{n+1}\right), \quad|\xi| \geqq M$. Finally $u_{0, b}^{\prime}(x, \lambda)$ may be shown by means of $(5.18 \mathrm{~b})$ and (6.2) to be of the form

$$
\text { (b) } u_{0, b}^{\prime}(x, \lambda)=y_{0}^{\prime}(x, \lambda)+e^{-(1 / 2) \xi \xi k-1 / 4} O\left(1 / \lambda^{n}\right), \quad|\xi| \geqq M .
$$

Consider next the situation when $x$ is on the range $\left(0, x_{+}\right)$. The equation (6.1) may be written in the form 
(a) $u_{0, b}(x, \lambda)=f_{1}(x, \lambda)+\int_{x_{+}}^{x} \Delta\left(y_{0}, y_{1}\right) \frac{\Omega(t, \lambda) e^{-k \pi i}}{\lambda^{n+1}(2 \lambda)^{1 / 2}} u_{0, b}(t, \lambda) d t$ wherein

(b) $f_{1}(x, \lambda)=y_{0}(x, \lambda)+\int_{b}^{x_{+}} \Delta\left(y_{0}, y_{1}\right) \frac{\Omega(t, \lambda) e^{-k \pi i}}{\lambda^{n+1}(2 \lambda)^{1 / 2}} u_{0, b}(t, \lambda) d t$.

In (7.6) one has already an estimate of $u_{0, b}(t)$ on the range $\left(x_{+}, b\right)$. Consequently the integral in $(7.7 \mathrm{~b})$ may be evaluated directly. Since $x$ is on an interval in which $\xi(x)$ is bounded, $y_{0}(x)$ and $y_{1}(x)$ are bounded; $y_{0}(t)$ and $y_{1}(t)$, on the other hand, are of the form (7.5). Thus

$$
\begin{aligned}
\Delta\left(y_{0}, y_{1}\right) u_{0, b}(t) & =\xi^{-1 / 2}(t)\left[O(1)+e^{-\xi(t)} \xi^{2 k}(t) O(1)\right] \\
& =\xi^{-1 / 2}(t) O(1),
\end{aligned}
$$

so that the integral is of $O\left(1 / \lambda^{n+1}\right)$. Hence

$$
\begin{aligned}
f_{1}(x, \lambda) & =y_{0}(x, \lambda)+O\left(1 / \lambda^{n+1}\right) \\
& =O(1) .
\end{aligned}
$$

In the integral of (7.7a) on the other hand, both $y_{\nu}(x)$ and $y_{v}(t)$ are bounded and so

$$
\int_{x_{+}}^{x}\left|\Delta\left(y_{0}, y_{1}\right) \frac{\Omega(t, \lambda) e^{-k \pi i}}{\lambda^{n+1}(2 \lambda)^{1 / 2}}\right| d t=O\left(1 / \lambda^{n+2}\right) .
$$

Equation (7.7a) is therefore seen to satisfy the hypothesis of the first form of the lemma, establishing that $u_{0, b}=f_{1}+O\left(1 / \lambda^{n+1}\right)$, and so

$$
\text { (a) } u_{0, b}(x, \lambda)=y_{0}(x, \lambda)+O\left(1 / \lambda^{n+1}\right), \quad|\xi| \leqq M .
$$

The equation (6.2) for $u_{0, b}^{\prime}(x, \lambda)$ may be written in a manner analogous to (7.7). Since $y_{0}^{\prime}(x)$ is $O\left(\lambda^{1 / 2}\right)$, it follows that

$$
\text { (b) } u_{0, b}^{\prime}(x, \lambda)=y_{0}^{\prime}(x, \lambda)+O\left(1 / \lambda^{n+1 / 2}\right), \quad|\xi| \leqq M .
$$

II. A solution which is dominant on (7.4). Consider that solution of the given equation which is designated by $u_{1,0}(x, \lambda)$, and which accordingly is represented by the integral equation (6.1) when $y=y_{1}$ and $x_{0}=0$ in that equation. The roles of $y$ and $\tilde{y}$ in the integrand are again filled by $y_{0}$ and $y_{1}$ respectively.

When $0 \leqq x \leqq x_{+}$, the situation is precisely that which was discussed in $\S 6$, and so

$$
\begin{aligned}
& \text { (a) } u_{1,0}(x, \lambda)=y_{1}(x, \lambda)+O\left(1 / \lambda^{n+2}\right), \\
& \text { (b) } u_{1,0}^{\prime}(x, \lambda)=y_{1}^{\prime}(x, \lambda)+O\left(1 / \lambda^{n+3 / 2}\right), \quad|\xi| \leqq M .
\end{aligned}
$$

When, on the other hand, $x_{+} \leqq x \leqq b$, the integral equation (6.1) is to be written in the form 
(a) $u_{1,0}(x, \lambda)=f_{2}(x, \lambda)+\int_{x_{+}}^{x} \Delta\left(y_{0}, y_{1}\right) \frac{\Omega(t, \lambda) e^{-k \pi i}}{\lambda^{n+1}(2 \lambda)^{1 / 2}} u_{1,0}(t, \lambda) d t$ in which

(b) $f_{2}(x, \lambda)=y_{1}(x, \lambda)+\int_{0}^{x_{+}} \Delta\left(y_{0}, y_{1}\right) \frac{\Omega(t, \lambda) e^{-k \pi i}}{\lambda^{n+1}(2 \lambda)^{1 / 2}} u_{1,0}(t, \lambda) d t$.

The integral in (b) may be estimated directly using (7.9a). In the integral only $y_{1}(x)$ is unbounded, so that

$$
\begin{aligned}
f_{2}(x, \lambda) & =y_{1}(x, \lambda)+e^{(1 / 2) \xi \xi-k-1 / 4} O\left(1 / \lambda^{n+2}\right) \\
& =e^{(1 / 2) \xi \xi-k-1 / 4} O(1) .
\end{aligned}
$$

In the integrand of (7.10a), on the other hand, both

$$
|\xi(x)| \geqq M \quad \text { and }|\xi(t)| \geqq M .
$$

Thus

$$
\begin{aligned}
e^{-(1 / 2)[\xi(x)-\xi(t)]}\left[\frac{\xi(x)}{\xi(t)}\right]^{k+1 / 4} \Delta\left(y_{0}, y_{1}\right) & =\xi^{-1 / 2}(t)\left[e^{\xi(t)-\xi(x)}\left[\frac{\xi(x)}{\xi(t)}\right]^{2 k} O(1)+O(1)\right] \\
& =\xi^{-1 / 2}(t) O(1),
\end{aligned}
$$

the second equality following because in this case $x>t$. The integral which corresponds to (7.1b) is thus of the order $O\left(1 / \lambda^{n+1}\right)$, so that, by the second statement of the lemma,

$$
\text { (a) } u_{1,0}(x, \lambda)=y_{1}(x, \lambda)+e^{(1 / 2) \xi \xi-k-1 / 4} O(1 / \lambda)^{n+1} \text {, }
$$

while from (6.2)

$$
\text { (b) } u_{1,0}^{\prime}(x, \lambda)=y_{1}^{\prime}(x, \lambda)+e^{(1 / 2) \xi \xi-k-1 / 4} O\left(1 / \lambda^{n}\right), \quad|\xi| \geqq M .
$$

The wronskian of the pair of solutions $u_{0, b}(x, \lambda)$ and $u_{1,0}(x, \lambda)$, being independent of $x$, may be evaluated at $x=0$ by means of (7.8), (7.9), and (5.14). When $|\lambda|>N$ and $\arg \lambda$ is on the interval $(-\pi / 2, \pi / 2)$,

$$
\text { wron }\left[u_{0, b}, u_{1,0}\right]=\operatorname{wron}\left[y_{0}, y_{1}\right]+O\left(1 / \lambda^{n+1 / 2}\right) \text {, }
$$

$O\left(1 / \lambda^{n+1 / 2}\right)$ being independent of $x$. Thus $u_{0, b}$ and $u_{1,0}$ are linearly independent.

8. Solutions appropriate to other configurations. It is clear that the discussion of preceding sections can be adapted to apply to any configuration where $x>0$ and where $\arg \lambda$ (which in this case equals arg $\xi$ ) is such that a certain $y_{\nu}$ is of subdominant form while an adjacent $y_{\mu}$ is dominant. A solution of the given equation is associated with $y_{\nu}$ by (6.3), taking $x_{0}=b$, and another with $y_{\mu}$ taking $x_{0}=0$. The deviation of each $u$ from the corresponding $y$ is then computed by a slightly modified version of the argument presented above. These results are summarized in the following theorem. 
Theorem 1. When $x>0,|\lambda|>N$, and $\lambda$ is in the sector $S_{\nu}(\lambda)$ :

(8.1) or

(a) $\quad(\nu-1 / 2) \pi \leqq \arg \lambda \leqq(\nu+1 / 2) \pi \quad$ if $\Re[\sigma \kappa] \leqq 0$,

(b) $(\nu-1 / 2) \pi+\epsilon \leqq \arg \lambda \leqq(\nu+1 / 2) \pi-\epsilon$ if $\Re[\sigma \kappa]>0$. $\nu=0,1,2$, or 3 ,

then $y_{v}(x, \lambda)$ is of subdominant form for large $\xi$, and

(a) $u_{\nu, b}(x, \lambda)=y_{\nu}(x, \lambda)+O\left(1 / \lambda^{n+1}\right) \quad$ when $|\xi| \leqq M$,

(b) $u_{\nu, b}(x, \lambda)=y_{\nu}(x, \lambda)+e^{-(1 / 2) \sigma \xi \xi \sigma k-1 / 4} O\left(1 / \lambda^{n+1}\right), \quad|\xi| \geqq M$.

Also

(a) $u_{\nu, b}^{\prime}(x, \lambda)=y_{\nu}^{\prime}(x, \lambda)+O\left(1 / \lambda^{n+1 / 2}\right) \quad$ when $|\xi| \leqq M$,

(b) $u_{\nu, b}^{\prime}(x, \lambda)=y_{\nu}^{\prime}(x, \lambda)+e^{-(1 / 2) \sigma \xi \xi \sigma k-1 / 4} O\left(1 / \lambda^{n}\right), \quad|\xi| \geqq M$.

On the remainder of the sector $\Sigma_{\nu}(\lambda)$ :

$$
(\nu-3 / 2) \pi+\epsilon \leqq \arg \lambda \leqq(\nu+3 / 2) \pi-\epsilon,
$$

$y_{\nu}(x, \lambda)$ is of dominant form and

(a) $u_{\nu, 0}(x, \lambda)=y_{\nu}(x, \lambda)+O\left(1 / \lambda^{n+2}\right) \quad$ when $|\xi| \leqq M$,

(b) $u_{\nu, 0}(x, \lambda)=y_{\nu}(x, \lambda)+e^{-(1 / 2) \sigma \xi \xi \sigma k-1 / 4} O\left(1 / \lambda^{n+1}\right), \quad|\xi| \geqq M$.

Also

(a) $u_{v, 0}^{\prime}(x, \lambda)=y_{\nu}^{\prime}(x, \lambda)+O\left(1 / \lambda^{n+3 / 2}\right) \quad$ when $|\xi| \leqq M$,

(b) $u_{\nu, 0}^{\prime}(x, \lambda)=y_{\nu}^{\prime}(x, \lambda)+e^{-(1 / 2) \sigma \xi \xi \sigma k-1 / 4} O\left(1 / \lambda^{n}\right)$.

On the intersection of the sectors $S_{\nu}(\lambda)$ and $\Sigma_{\mu}(\lambda)$, where $\mu$ is adjacent to $\nu$ (i.e. $\mu-\nu$ is an odd integer), the functions $u_{\nu, b}(x, \lambda)$ and $u_{\mu, 0}(x, \lambda)$ are linearly independent, with wronskian given on this sector by

$$
\text { wron }\left[u_{\nu, b}, u_{\mu, 0}\right]=\text { wron }\left[y_{\nu}, y_{\mu}\right]+O\left(1 / \lambda^{n+1 / 2}\right) \text {. }
$$

Slight modifications are necessary when $x<0$ since in this case arg $\xi$ $=2 \pi+\arg \lambda$, and the interval terminates at $a$ instead of $b$. The function subdominant on the sector $S_{\nu}(\lambda)$ is opposite to $y_{\nu}$, hence is $y_{\tau}(x, \lambda)$ with $\tau=\nu+2$ modulo 4 , and the corresponding solution of the given equation is $u_{r, a}(x, \lambda)$.

THEOREM 2. The statement of Theorem 1 becomes correct for $x<0$ if the functions $y_{\nu}, u_{\nu, b}$ and $u_{\nu, 0}$ are everywhere replaced by $y_{\tau}, u_{\tau, a}$, and $u_{\tau, 0}$ respectively, with $\tau=\nu+2$ modulo 4 .

When neither of the numbers $\kappa \pm 1 / 4$ is an integer it proves possible to obtain a pair of independent solutions of the given equation which, on the sector $S_{\nu}(\lambda)$, are asymptotically distinguishable over the entire interval $(a, b)$. The functions in question are $u_{v, b}$ and $u_{\tau, a}$. It has already been shown that 
when $|\xi| \geqq M$ these solutions are of subdominant form when $x>0, x<0$ respectively. The wronskian may be computed from Theorems 1 and 2 by setting $x=0$ in the formulas given there. It is found that on the sector $S_{\nu}(\lambda)$

$$
\text { wron }\left[u_{\nu, b}, u_{r, a}\right]=\text { wron }\left[y_{\nu}, y_{\tau}\right]+O\left(1 / \lambda^{n+1 / 2}\right) \text {, }
$$

and so $u_{\nu, b}$ and $u_{\tau, a}$ are linearly independent. $u_{\tau, a}$ is of course a linear combination of the independent pair of functions $u_{\nu, b}$ and $u_{\mu, 0}$ ( $\mu$ being adjacent to $\nu$ ). Hence

$$
\text { (a) } u_{r, a}=c_{1} u_{v, b}+c_{2} u_{\mu, 0}
$$

with coefficients given by the known formulas

$$
\text { (b) } c_{1}=\frac{\operatorname{wron}\left[u_{\tau, a}, u_{\mu, 0}\right]}{\operatorname{wron}\left[u_{v, b}, u_{\mu, 0}\right]}, \quad c_{2}=\frac{\operatorname{wron}\left[u_{v, b}, u_{\tau, a}\right]}{\operatorname{wron}\left[u_{v, b}, u_{\mu, 0}\right]} \text {. }
$$

It is easy to calculate, with the use of (5.19), that these coefficients differ from those in the corresponding formulas between the functions $y$ by quantities of the order $O\left(1 / \lambda^{n+1}\right)$. The latter formulas have already been derived; they are essentially the equations (5.14). If therefore when $x>0$ the formulas for $u_{v, b}$ and $u_{\mu, 0}$ in terms of $y_{\nu}$ and $y_{\mu}$ are substituted into (8.9), it is seen that $u_{\tau, a}$ differs from $y_{\tau}$ on this interval by a function $O\left(1 / \lambda^{n+1}\right) \cdot y_{\nu}+O\left(1 / \lambda^{n+1}\right) y_{\mu}$. Of course when $x<0$ the roles of $\nu$ and $\tau$ are reversed. The results are summarized below.

THEOREM 3. If neither of the numbers $\kappa \pm 1 / 4$ is an integer, then when $|\lambda|>N$ the functions $y_{\nu}(x, \lambda)$ and $y_{\tau}(x, \lambda)$, with $\tau=\nu+2(\bmod 4)$, are linearly independent and $y_{\nu}$ is of subdominant form. The corresponding solutions $u_{\nu, b}$ and $u_{\tau, a}$ of the given differential equation are likewise linearly independent on the sector $S_{\nu}(\lambda)$ and have there the form

(a) $u_{p, b}(x, \lambda)=y_{p}(x, \lambda)+e^{-(1 / 2) \sigma \xi \xi \sigma k-1 / 4} O\left(1 / \lambda^{n+1}\right)$,

(b) $u_{\tau, a}(x, \lambda)=y_{\tau}(x, \lambda)+e^{(1 / 2) \sigma \xi \xi-\sigma k-1 / 4} O\left(1 / \lambda^{n+1}\right)$,

these formulas valid when $|\xi| \geqq M$ and $x>0$.

When $x<0$ and $|\xi| \geqq M, y_{\nu}$ and $y_{\tau}$ again are independent of the sector $S_{\nu}(\lambda)$, with $y_{\tau}$ subdominant. The related solutions $u$ are of the form

$$
\begin{aligned}
& \text { (a) } u_{\nu, b}(x, \lambda)=y_{r}(x, \lambda)+e^{(1 / 2) \sigma \xi \xi \sigma k-1 / 4} O\left(1 / \lambda^{n+1}\right), \\
& \text { (b) } u_{\tau, a}(x, \lambda)=y_{\tau}(x, \lambda)+e^{-(1 / 2) \sigma \xi \xi \sigma k-1 / 4} O\left(1 / \lambda^{n+1}\right),
\end{aligned}
$$

the formulas being valid when $|\xi| \geqq M$ and $x<0$.

Finally, on $S_{\nu}(\lambda)$ when $|\xi| \leqq M$,

$$
\begin{aligned}
& \text { (a) } u_{r, b}(x, \lambda)=y_{\nu}(x, \lambda)+O\left(1 / \lambda^{n+1}\right), \\
& \text { (b) } u_{\tau, a}(x, \lambda)=y_{\tau}(x, \lambda)+O\left(1 / \lambda^{n+1}\right),
\end{aligned}
$$

these formulas being independent of the sign of $x$. 


\section{REFERENCES}

1. G. D. Birkhoff, On the asymptotic character of the solutions of certain linear differential equations containing a parameter, Trans. Amer. Math. Soc. vol. 9 (1908) pp. 219-231.

2. R. L. Evans, Solution of linear ordinary differential equations containing a parameter, Final report, Office of Ordnance Research Contract DA-11-022-ORD-489 with the University of Minnesota, September, 1953.

3. R. E. Langer, The asymptotic solutions of ordinary linear differential equations of the second order, with special reference to a turning point, Trans. Amer. Math. Soc. vol. 67 (1949) pp. 461-490.

4. - The asymptotic solutions of certain linear ordinary differential equations of the second order, Trans. Amer. Math. Soc. vol. 36 (1934) pp. 90-106.

5. E. T. Whittaker and G. N. Watson, Modern analysis, 2d ed., Cambridge, 1915.

UNIVERSITY OF WISCONSIN,

Madison, Wis. 